\section{ON A RATIO TEST OF FRINK}

BY

\section{STARK (WROCEAW)}

In a recent paper ${ }^{1}$ ) Frink gave the following test for con vergence of a series of positive terms:

If for some positive integer $k$

$$
\varlimsup_{n \rightarrow \infty}\left(\frac{a_{n}}{a_{n-k}}\right)^{n}<e^{-k}
$$

then the series is convergent; if for some $k$

$$
\left(\frac{a_{n}}{a_{n-k}}\right)^{n} \geqslant e^{-k} \text { for } n>N
$$

then the series is divergent.

It is interesting to notice that the inequality ( 1 ) is equivalent (for each positive integer $k$ ) to the inequality

$$
\varlimsup_{n \rightarrow \infty} n\left(\frac{a_{n}}{a_{n-k}}-1\right)<-k,
$$

i. e. from (1) follows (3), and conversely. For $k=1$ it follows that the series satisfying Frink's test of convergence are identical with those satisfying the test of Raabe.

Indeed, suppose (1). Then

$$
\varlimsup_{n \rightarrow \infty}\left(\frac{a_{n}}{a_{n-k}}\right)^{n}<e^{-a}<<e^{-k},
$$

$s>k$.

Hence

$$
\begin{array}{cc}
\left(\frac{a_{n}}{a_{n-k}}\right)^{n}<\left(1-\frac{1}{n}\right)^{n s}, & n>N_{1} ; \\
\frac{a_{n-k}}{a_{n}}>\left(\frac{n}{n-1}\right)^{s}=\left(1+\frac{1}{n-1}\right)^{s} \geqslant 1+\frac{s}{n-1}, & n>N_{1} ;
\end{array}
$$

1) O. Frink, A ratio test, Bulletin of the American Mathematical Society 55 (1948), p. 953.

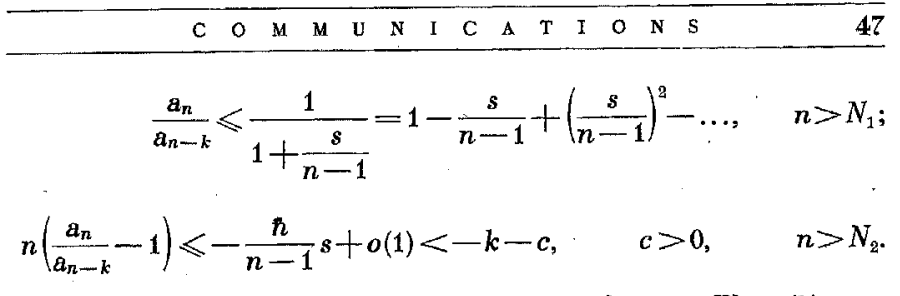

The converse implication is still more obvious. Thus (3) may be used instead of (1) as a convergence test. It may be proved independently from Frink's reasoning and equally easily, on the usual lines of proving Raabe's test by comparing $\sum \mathrm{a}_{n}$ with $\sum n^{-1}$.

As for the divergence test (2) it is easy to follow from (2) the inequality

$$
n\left(\frac{a_{n}}{a_{n-k}}-1\right) \geqslant-k, \quad n>N_{3},
$$

but not conversely, because (4) is equivalent to

$$
\left(\frac{a_{n}}{a_{n-k}}\right)^{n} \geqslant\left(1-\frac{k}{n}\right)^{n}, \quad n>N_{s} .
$$

Therefore, if a series satisfies the divergence test of Frink, it satisfies the generalized divergence test (4) of Raabe, but not conversely.

For instance, take the harmonic series

$$
k=1, \quad a_{1}=1, \quad a_{n}=a_{n-1}\left(1-\frac{1}{n}\right) .
$$

May, 1949. 